\title{
Liver ischemia and reperfusion injury. Pathophysiology and new horizons in preconditioning and therapy ${ }^{1}$
}

Paula Carolina Grande Nakazato', João Paulo Victorino", Clarice Fleury Final', Karina Dal Sasso Mendes ${ }^{\prime v}$, Maria Cecília Jordani Gomes"II, Paulo Roberto Barbosa Evorav, Luiz Augusto Carneiro D’Albuquerque ${ }^{\mathrm{vI}}$, Orlando Castro-e-Silva ${ }^{\mathrm{VII}}$

'Medical student, Surgery and Anatomy Department, Medical School, Universidade de São Paulo (USP), Ribeirao Preto$\mathrm{SP}$, Brazil. Scientific and intellectual content of the study; acquisition, analysis and interpretation of data.

"BSCN, USP, College of Nursing, PAHO/WHO Collaborating Centre for Nursing Research Development, Ribeirao Preto-SP, Brazil. Scientific and intellectual content of the study, manuscript preparation.

"'Laboratory Specialist, Surgery and Anatomy Department, Medical School, USP, Ribeirao Preto-SP, Brazil. Scientific and intellectual content of the study.

IVPD, General and Specialized Nursing Department, USP, College of Nursing, PAHO/WHO Collaborating Centre for Nursing Research Development, Ribeirao Preto-SP, Brazil. Scientific and intellectual content of the study.

${ }^{\vee}$ Full Professor, Surgery and Anatomy Department, Medical School, USP, Ribeirao Preto-SP, Brazil. Scientific and intellectual content of the study.

${ }^{V}$ 'Full Professor, Department of Gastroenterology, Medical School, USP, Ribeirao Preto-SP, Brazil. Scientific and intellectual content of the study.

VIIFull Professor, Surgery and Anatomy Department, and Department of Gastroenterology, Medical School, USP, Ribeirao Preto-SP, Brazil. Scientific, intellectual, conception and design of the study; acquisition, analysis and interpretation of data; manuscript writing; critical revision; final approval.

\section{Abstract}

It is well known that during hepatic operative procedures, it is often critical that the irrigation is interrupted to avoid possible bleeding, blood transfusions, variable intensities, and their short and long-term consequences. It was believed in the past that the flow interruption should not exceed 20 minutes, which limited the use of this maneuver. However, it has been postulated that ischemia could be maintained for more than 60 minutes in healthy livers. The present paper review includes: 1) A brief introduction to justify the rationale of the review design; 2) Aspects of the pathophysiology of the three stages of the liver ischemia-reperfusion injury; 3) The innate and acquired immunity; 4) Oxidative stress; 5) Apoptosis and autophagy, Some essential biomarkers (Tumor Necrosis Factor- $\alpha$, nitric oxide, metalloproteinases); and, finally; 6) Preventive ("cheating") strategies, non-pharmacological and pharmacological options to treat the liver IR injury.

Key words: Liver. Ischemia. Reperfusion Injury 


\section{- Introduction}

It is well known that during hepatic operative procedures, it is often critical that the irrigation is interrupted to avoid possible bleeding, blood transfusions, variable intensities, and their short and long-term consequences. A series of maneuvers are used to do this, the best known being the Pringle maneuver, or clamping of the hepatic pedicle. Initially, the Pringle maneuver was described as an interruption of blood flow to the portal vein and hepatic artery by digital compression of the hepatic pedicle in the hepatoduodenal ligament ${ }^{1-3}$.

It was believed in the past that the flow interruption should not exceed 20 minutes, which limited the use of this maneuver. However, in subsequent studies, it has been postulated that ischemia could be maintained for more than 60 minutes in healthy livers ${ }^{1-3}$. Thus, reports with long periods of intermittent or continuous ischemia have been progressively reported, with excellent results. However, new techniques and adaptations of this maneuver emerge in clinical practice, such as the intermittent Pringle maneuver, the continuous Pringle maneuver, whose periods of ischemia and reperfusion are not standardized.

Also, standard experimental in training, is total vascular exclusion, achieved by clamping the portal triad, intrahepatic and suprahepatic vena cava, and selective vascular exclusion, which preserves the flow of the vena cava and disconnects the liver from the vena cava through connections with tiny perforating veins ${ }^{1-3}$.

Currently, the use of intermittent or continuous prolonged (more than 60 minutes) techniques are proven to be safe; however, the latter presented benefits as intraoperative hemorrhages and blood transfusions. The intermittent obstruction of the vascular flow can be safely extended for 120 minutes and has a maximum limit of 300 minutes in extreme cases. In steatotic livers, 120-minute ischemia remains safe and its time limit is 200 minutes $^{3}$.
On the other side, when blood supply is interrupted for an extended period and then reestablished, injury of ischemia-reperfusion (IR) occurs, which in turn may culminate in functional loss of the remaining tissue.

The IR lesion can be classified as warm (normothermic) or cold (hypothermic). The first form occurs during liver surgery, trauma and shock and the latter form during the preservation and storage of the organ in transplants. ERO is generated in both processes. However, the maintenance of oxidative stress differs between both types of ischemia. Concerning to metabolism and molecular pathways, warm ischemia is more damaging, because, in hypothermic ischemia, cellular metabolism and ATP consumption are significantly lower ${ }^{1-2,4}$.

It is apparently paradoxical that in steatotic livers, the I/R lesion tends to be even worse, depending on the degree of steatosis; the worst results are obtained in the moderate and severe cases of microvesicular steatosis. Thus, in steatosis, worsening of graft and receptor survival is observed in the case of transplants, with the increased histological lesion and hepatic functional derangement ${ }^{5-6}$.

The pathophysiology of liver ischemia and reperfusion injury are always evolutionary justifying, as clear rationale, the constant update to discuss new horizons in preconditioning and therapy.

\section{Aspects of pathophysiology}

Ischemia-reperfusion injury can lead to cytokines, cytokines, circulating chemokines and ROS, damage to organs and more distant tissues, such as kidneys, adrenals, intestines, pancreas, lungs, and myocardium, as well as the liver itself ${ }^{1,7}$.

\section{Early stage}

Deprivation of nutrients and oxygen, secondary to ischemia, will cause primary liver damage. As the availability of ATP is reduced, ATP-dependent ion channels begin to fail; cell 
metabolism slows down, anaerobic glycolysis is activated. Calcium ions accumulate within cells due to incompetence of the Ca channels. These ions activate intracellular enzymes such as phospholipase $C$, protein kinase $C$ and induce hepatic necrosis and apoptosis. The accumulation of lactic acid acidifies the medium, conferring protection to the mitochondrial membrane, in the first moment, and postpones the cell death. Already the increase of the $\mathrm{pH}$ seems to accelerate its death; is what is called the $\mathrm{pH}$ paradox. Later, $\mathrm{pH}$ imbalance and ion accumulation due to ion channel incompetence, lead to the transition of mitochondrial permeability, an essential effect for cell death (Figure 1).

This process is characterized by mitochondrial swelling, followed by reduction of the mitochondrial membrane potential and finally allows the passage of high molecular weight molecules (less than $1500 \mathrm{kDa}$ ) by the "ionic mega channels"8-10.

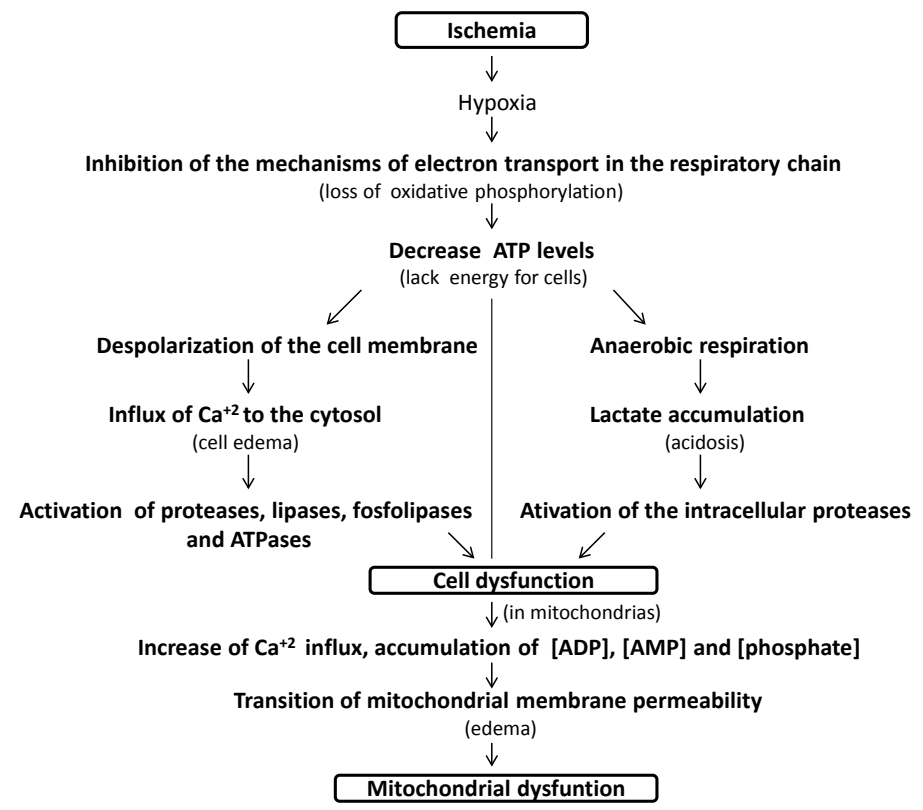

Figure 1 - Simplified scheme of cellular bioenergetic processes involved in ischemic injury. The oxygen depletion caused by the inhibition of the mechanisms of electron transport in the respiratory chain leads to a decrease in the cellular levels of ATP, originating $\mathrm{Na}+\mathrm{K}+$ failure and generating depolarization of the cell membrane with consequent intracellular accumulation of $\mathrm{Ca}^{+2}$, activating proteases, lipases, phospholipases and ATPases. At the same time, the change from aerobic to anaerobic metabolism causes lactic acidosis which also activates intracellular proteases that cause cell dysfunction. In relation to the mitochondria, the increase of $\mathrm{Ca}^{+2}$ influx to the organelle, associated to the accumulation of ADP, AMP and phosphate, leads to the transition of membrane permeability and mitochondrial dysfunction. These events can lead to cell death.

\section{Intermediate phase}

The dysfunctions caused by the initial phase will cause the formation of reactive oxygen species (ROS), which through peroxidation and chemotaxis will cause damage and death of adjacent cells, production of potent cytokines and chemokines, essential for the progression of the inflammatory response. Immune cells will be activated locally and systemically by mediators such as CK and CD4 T cells, and adhesion factors will promote wide neutrophil infiltration. As a consequence of this process, the microvasculature and sinusoidal endothelial cells will be compromised, worsening the tissue conditions. The pathophysiological mechanisms are detailed below ${ }^{8-9,11}$. 
Innate and acquired immunity

Once the cellular injury occurs, innate cellular immunity is activated. Pattern recognition receptors identify PAMP and DAMP present in the circulation. In the case of the IR lesion, six classes of standard recognition receptors (RRP) are current: Toll-like receptors and C-typelectin receptors, which are membrane receptors and RIG-I like receptors, NOD-like receptors, receptors AIM2-like receptors and oligoadenylate synthetase-like receptors, which are cytoplasmic receptors. Increased activation of Toll-like 4 receptors appears to be involved in the higher susceptibility of steatotic livers to IR injury. Hepatocytes and Kupffer cells (CK) have predominantly RTL2 3 and 4. In general, the activation of RRPs by DAMP triggers intracellular cascades that lead to transcription of chemokines and cytokines such as tumor necrosis factor (TNF) - $\alpha$, interleukins (IL) and Interferons (IF), ERO and other cytotoxic molecules $^{12-14}$ (Figure 2).

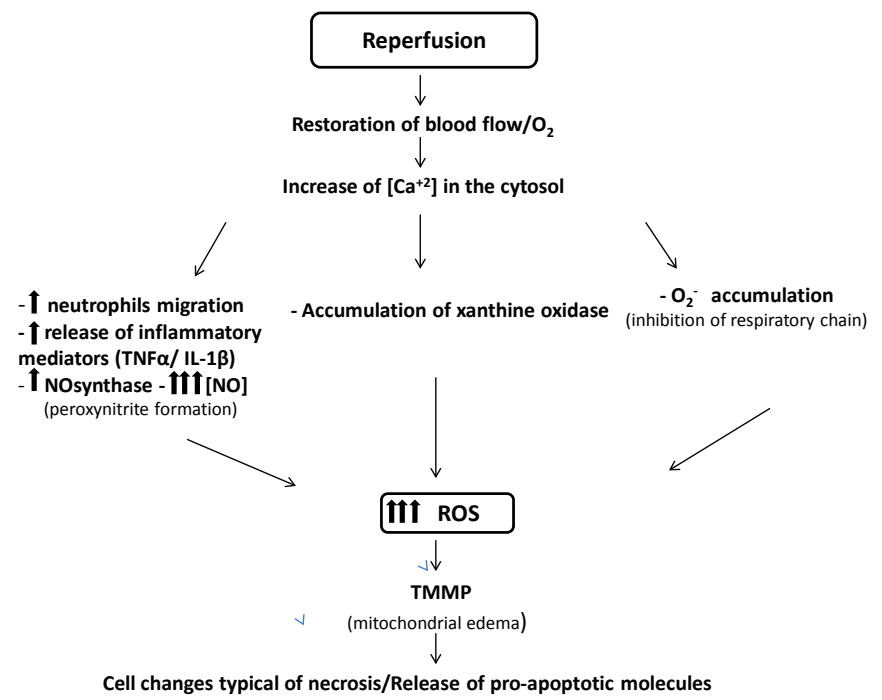

Figure 2 - Processes involved during the reperfusion Injury. Restoration of blood flow associated with increased intracellular $\mathrm{Ca} 2+$ concentration leads to the disordered increase of ROS by several pathways. This increase leads to cellular and mitochondrial changes that are often irreversible, leading to cell death.

In humans, approximately $10 \%$ of the intrahepatic lymphocytes are Natural Killer T cells (CTNK), directly related to various liver diseases, diabetes, lupus and even to tumor immune vigilance. CTNK seems to make the connection of the innate immune system with the adaptive immune system in various organs. In contrast to CD4 lymphocytes, CTNKs are easily activated; any antigen presenting cell expressing CD1d is capable of enabling them. Much of the liver cells express CD1d, thus, in the IR lesion, CTNKs become numerous and essential for the propagation of the inflammatory stimulus. These cells make up a heterogeneous group and can be roughly divided into type I, invariant, and CTNK type II, which harbor greater varieties of receptors than type I and have anti-inflammatory and limiting effects of tissue injury. Humans have, proportionally, a more significant amount of CTNK type II, so it is thought that these may be the primary agents in the immunomodulation of the lesion ${ }^{15}$.

Recruitment of T lymphocytes depends mainly on the release of chemokines, so it is a process of innate immunity and release of inflammatorymediators (Figure 2). Recruitment of T lymphocytes is dependent on two signals; the first occurs by the presentation of antigen to the $T$ cell receptors and the second signal 
is the co-stimulation by nonspecific molecules, generally related to antigen-presenting cells (APCs). These molecules are quite varied in function and patterns; among the most studied are CD40-CD154 and CD28 / CD80 / 86 16. In the first hour after reperfusion, before significant neutrophil accumulation, CD4 lymphocytes are present, but not CD8. Therefore, inactivation of CD4 decreases IL-17 secretion, suppressing neutrophil recruitment in these cases. IL17 induces the release of cytokines and chemokines by peritoneal mesothelium, epithelial cells, fibroblasts, osteoblasts and endothelial cells, thereby amplifying the inflammatory and chemotactic response. The relationship of T-cell recruitment and antigenic mechanisms has not yet been fully elucidated. Studies with detection of reactive IgM with damaged tissues present conflicting results with results from studies using MHC II antibodies to reduce IR lesion.

\section{Oxidative stress}

Reactive oxygen species (ROS) are produced during ischemia, but increase massively after reperfusion. Hydroxyl radicals, superoxide anions, and hydrogen peroxide contribute to mitochondrial dysfunction, causing chemotaxis, lipid peroxidation, destroying the integrity of proteins and membranes. Additionally, they may cause damage to endothelial cells and dysfunction in the microvasculature ${ }^{9,11}$.

Oxidative stress can have three primary sources: the electron chain, NADPH oxidase, and xanthine oxidase. During mitochondrial respiration, faults may occur in the electron chain, leading to the formation of superoxide anions, not necessarily related to pathological processes. In long periods of ischemia, mitochondrial dysfunction occurs, leading to a higher tendency to form ROS during mitochondrial respiration.

During ischemia, degradation of ATP culminates in the accumulation of intracellular hypoxanthine. Concomitantly, xanthine dehydrogenase (XD) is converted to xanthine oxidase (XO), which turns hypoxanthine to superoxide anion, hydrogen peroxide, and urate when the oxygen supply is reestablished. Thus, reperfusion enhances oxidative stress. In hypothermic IR lesions, the transformation of XD into $X O$ is slower in hepatocytes and sinusoidal endothelial cells. However, in the late phase of both hot and hypothermic IR lesions, CK are the primary sources of ROS. Steatosis reduces liver tolerance to IR injury and increases sensitivity to ROS. Hepatic steatosis rises lipid peroxidation by decreasing the availability of antioxidants and rising ROS production $^{16-17}$.

Late phase

In the later stages of the I/R lesion (224 hours after reperfusion), the primary source of ROS is neutrophils. Neutrophil migration depends on the chemotaxis, cytokines, chemokines, and adhesion factors initially released by CK. Selectin has synergistic actions: endothelial P-selectin and leukocyte L-selectin begin the rolling phase of leukocytes while E-selectin may not play a vital role in the process but contribute to T-cell adhesiveness and attraction. Selectin blockade confers protection to the liver during the IR lesion, although specific blockers for this class of molecules have not been clinically tested in surgeries. In addition to the selectins, integrins and immunoglobulins are expressed during reperfusion, contributing to the transmigration of neutrophils through sinusoidal endothelial cells ${ }^{18}$.

\section{Tumor Necrosis Factor- $\alpha$ (TNF- $\alpha$ )}

Upon activation, CKs release TNF- $\alpha$, a potent nonspecific primary inflammatory mediator. Its action is complementary to the extent that it induces both death and cellular regeneration. When binding to specific TNF- $\alpha$ receptors on the cell membrane, a series of intracellular reactions occur, the end of 
which is the activation of effector caspases 3 and 7, leading to apoptosis. Recent studies still demonstrate similarities of this apoptosis pathway with necrosis, in this case, called programmed necrosis or necroptosis. Binding of TNF- $\alpha$ to its membrane receptor may even induce the encoding of transcription factors and NF-KB, which in turn will lead to cell proliferation. The release of interleukin-1 and interleukin-6 is enhanced, as well as other adhesion factors, such as ICAM-1, VCAM1 , and P-selectin. Different cell types such as neutrophils, NK, and CD4 lymphocytes will release TNF- $\alpha$ later in life. The functions of this mediator, although opposite, are complementary. Thus, its complete inhibition is not desirable to maintain hepatic proliferative capacity ${ }^{19}$ (Figure 2 ).

\section{Nitric Oxide (NO)}

Endothelial cells, due to the influx of calcium, produce endothelial nitric oxide synthase (eNOS). This enzyme will allow the formation of NO, mainly in the initial stages of ischemia. Inflammatory cells and hepatocytes induce the expression of inducible nitric oxide synthase (iNOS), the enzyme responsible for most of the NO produced in the IR lesion. Interleukin-1 and TNF- $\alpha$ will be accountable for increasing iNOS expression later. Additionally, $\mathrm{NO}$ is an important inflammatory mediator; it suppresses the production of IL-1, -12, TNF- $\alpha$ and the activation of helper $\mathrm{T}$ lymphocytes; increases production of regulatory Th2 and $\mathrm{T}$ cells and controls leukocyte adhesiveness. The protective function of NO against IR injury is complex and comprises several aspects. Microcirculation improves, and the availability of high energy folate increases in the presence of NO. Furthermore, the expression of adhesion molecules, such as ICAM-1, selectins, endothelin-1 and p53 gene is significantly decreased by NO. ERO production also declines, since NO inhibits mitochondrial cytochrome oxidation, and therefore, the electron chain.
When activated by NO, soluble guanylyl cyclase (sGC), guanosine triphosphate (GTP) is catalyzed in cyclic guanosine monophosphate. This pathway determines intracellular reactions responsible for protection against cell death in hepatocytes. However, NO in large amounts has deleterious effects. Thus, inhibition of iNOS / NOS overexpression exerts anti-inflammatory and antioxidant effects. Preconditioning can be used for the protective effects of NO; being essential, for this, the control of its production, so that it does not reach high levels ${ }^{9,20}$.

\section{Matrix Metalloproteinases (MPM)}

Both hepatocytes, star cells, CK and, sinusoidal endothelial cells secrete, during the IR lesion, MPM, tissue architecture remodeling enzymes. Its expression determines the recruitment of stellate cells and consequent fibrosis of the organ, so when exacerbated, prolonged it is harmful. MPM-2 and -9 (respectively gelatinases $A$ and $B$ ) are central to the lesion, considering its involvement with degradation with collagen IV and fibronectin. Experiments with MPM inhibitors showed a significant reduction of the $\mathrm{I} / \mathrm{R}$ lesion and the release of cytokines; however, there are indications that the use of anti-MMP monoclonal antibodies to achieve MPM- 9 is even more effective. However, although the lesion is aggravated by MPM-9, the absence of this enzyme delays hepatic recovery in later periods (24 to 72 hours) after reperfusion. In addition to remodeling function, MPM can release growth factors from the extracellular matrix, membrane surfaces and latent proteins $^{21}$.

Cell death

When the cell passes through the IR lesion and ceases to be viable, two processes can occur: apoptosis and necrosis. Because it is a programmed, regulatory death, apoptosis does not show extravasation of intracellular components nor intensify inflammation. In contrast, necrosis alters the morphology of 
cellular organelles, presents cellular edema and ruptures of membranes with extravasation of intracellular contents. This content contributes to the inflammation and aggression of adjacent healthy tissues. In the TUNEL examination, the necrotic cells color, passing the idea that apoptosis in IR is the majority. Morphological analyzes indicate that, in reality, necrosis, also called oncosis, is more intense and responsible for aggravating the inflammatory process and injury.

The transition of mitochondrial membrane permeability (TPMM) is directly related to necrosis, as it is followed by the opening of pores and ion channels that cause mitochondrial decoupling, ATP depletion and loss of membrane potential. When a small number of mitochondrial pores open, mitochondrial destabilization followed by necrosis still occurs. In this sense, the increase of intracellular calcium is an aggravating factor: it induces the formation of protein kinase $C$, indirectly activates the NF-kB to increase the production of ROS and facilitates the TPMM. Mitochondrial membrane dysfunction soon activates apoptotic cascades. Derivatives of the mitochondria rupture, pro-apoptotic proteins are released into the intracellular medium, which induces the formation of cytochrome C, apoptosis inducible factor, components and critical inducers of the apoptosome. DIABLOSmac, when released from the interior of the mitochondria, inhibits caspase inhibitors, inducing apoptosis ${ }^{22}$.

\section{Autophagy}

Autophagy is described in the literature as a process related to both cell death and hepatocellular survival by the degradation of intracellular compounds to maintain ATP production and remove damaged organelles and aggregates of proteins. Autophagy can provide an additional period before cell death, providing therapies aimed at reducing the extent of damage. Episodes of severe ischemia can cause an increase in the occurrence of autophagy, which leads to the destruction of intracellular proteins and organelles, resulting in necrosis or apoptosis. Some results of autophagy studies in the IR lesion are still discordant due to different methods and analyzes; leaving gaps in the correlation of autophagy with the IR lesion ${ }^{23}$.

\section{Cheating the IR injury}

To mitigate the lesion caused and prevent organ loss, various strategies have been studied, from surgical interventions, pharmacological agents to gene therapy. Several techniques of selective or total occlusion are proposed to reduce hemorrhage, but, in general, they present negative results about IR. In the literature, the reversal of the mitochondrial lesion in the 20-minute ischemia has been described; the groups were evaluated immediately and 20 minutes after reperfusion. Improvement in mitochondrial respiration and morphological recovery directly related to the type and intensity of the lesion ${ }^{24}$, were observed. The surgical technique also includes hypothermic perfusion and parenchymal hypothermia, capable of reducing the inflammatory response and increasing ischemic tolerance ${ }^{25}$. IR reduction strategies, modulation of the renin-angiotensin-aldosterone system (RAAS), chemokines, oxidative stress, CK and leukocyte activity; as well as the minimization of hepatic steatosis. Inhibition of RAAS, by NO suppression, reduces oxidative stress; the test with angiotensin receptor antagonists promoted lower cell death and a higher survival rate. With regard to inflammatory modulators, corticosteroids were able to reduce acute injury and postoperative complications while the elimination of $\mathrm{CK}$ and administration of protease inhibitors improved the viability of the grafts and prevented reperfusion injury ${ }^{26}$. Similarly, adenosine cumulative extracellular activity has global anti-inflammatory action in hypoxia and injury. The adenosine-ADORA 
signaling pathways appear to reduce reperfusion injury and improve hepatocellular function; in a human organ transplant, may be a potential tool ${ }^{27}$.

Conditionalities, pharmacological options, and emerging options

Described initially in the heart and kidneys, ischemic preconditioning (ICP) consists of short periods of ischemia followed by reperfusion that give the organ more excellent resistance to prolonged periods of ischemia. The mechanisms of ICP remain unclear, although some authors attribute their protective action to the reduction in TNF- $\alpha$ levels of CK, associated with increased adenosine monophosphate kinase. In this way, the availability of ATP would increase, reducing the effects of its depletion. Experimental results show a reduction of ALT and AST enzymes and an increase in the Respiratory Control Ratio (RCR) index, which reflects the preservation of mitochondrial function in the groups that passed the $\mathrm{PCl}$ about those that did not pass ${ }^{28-29}$. In studies performed with cirrhotic livers, the parameters remained similar between the cirrhotic groups, with $\mathrm{IR}$ or $\mathrm{PCl}$ induction ${ }^{30}$. Recent studies confirm the efficacy of the process in reducing hepatocellular injury, but the results are discrepant due to the lack of standardization and the variability in the time of ischemia, which reinforces the need for a protocol to allow the comparison between studies ${ }^{31-32}$. During $\mathrm{PCl}$, the inducible factor of hypoxia-1 $\alpha$ (HIF-1 $\alpha$ ), a substance directly related to hepatocellular protection, is induced. HIF-1 seems to influence revascularization and may promote graft regeneration, leading to improved function and survival rate. Under physiological conditions, HIF-1 is easily degraded during normoxia and reoxygenation. Therefore, it is not efficient to attenuate the lesion ${ }^{33}$.

On the other hand, ischemic postconditioning (PosCl) consists of brief periods of reperfusion before prolonged final reperfusion. The mechanisms that involve $\mathrm{Pos} \mathrm{Cl}$ resemble in many respects the CIP. In recent studies, a decrease in serum ALT and AST levels, preservation of superstructure integrity and mitochondrial function, and a decrease in apoptosis rates and NF-kB expression have been observed in recent studies. The authors attributed these results to the controlled episodes of reperfusion; antiapoptotic proteins would be expressed in greater quantity in these cases. Other studies showed a considerable presence of NO, iNOS and serum eNOS in tissues submitted to PosCl, justifying another protective pathway. The comparison between parameters of $\mathrm{PCl}$ and PosCl groups did not demonstrate significant differences in warm ischemia: the reduction in ALT and AST, apoptosis rate, functional preservation and survival rate corresponded. In hypothermic ischemia, on the other hand, $\mathrm{PosCl}$ presented more effective results than $\mathrm{PCl}$. The associations of the two processes presented synergistic action in comparison with $\mathrm{PCl}$ or isolated PosCl. ALT and AST decrease while antioxidant activity, hypoxia tolerance and cell proliferation increase; however, regarding the release of cytokines, there was no difference about the conditions alone $e^{34}$.

In steatotic livers submitted to $\mathrm{PCl}$, there was a reduction in histological damage of ALT and AST levels; better preservation of liver function and better survival, assigning a certain degree of protection. The studies with steatotic livers are quite heterogeneous as a technique and duration of ischemia, form of induction of steatosis, certain results, fact that limit the conclusions. In studies with hypothermic ischemia lasting more than 6 hours, $\mathrm{PCl}$ had minimal results, generally ${ }^{31}$.

\section{Pharmacological interventions}

There are many inhibit inflammatory mechanisms and mediators in various ways that 
allow several pharmacological interventions. However, there are no adequate and sufficient clinical research and studies, their use remains limited and permeated by uncertainties. It is known that prednisolone has antiinflammatory action and can reduce cell death, inflammatory markers, possible postoperative complications and length of the hospitalization. Pharmacological post-conditioning (PCF) seeks to attenuate IR injury through administration of agents during reperfusion. The use of drugs such as milrinone, diazoxide, agonists of A2A receptors administered at peri-reperfusion times showed reduced levels of ALT, AST, $\mathrm{CDH}$ and cell death, reflecting hepatocellular protection ${ }^{1,5}$. Antioxidants also comprise a form of preconditioning, because by reducing the ROS produced by the different cells, they attenuate the harmful responses; compounds such as glutathione, superoxide dismutase, catalase are present in large amounts in the liver, offering it an admirable resistance. In this line, the possibility arises of the use of natural antioxidants, phyto-antioxidants, and stimulation of the internal antioxidant signaling system.

Among the pharmacological options, treatments are developed to reduce the IR lesion in liver transplants. Methylprednisolone, pan-caspase inhibitors, recombinant P-selectin glycoprotein, $\mathrm{N}$-acetylcysteine linkers have already undergone clinical studies and, except $\mathrm{N}$-acetylcysteine, all have short-term survival benefits. Methylprednisolone was able to reduce chemokine levels in donor tissues, as well as levels of ALT and AST, reflecting preservation of the graft. Pan-caspase inhibitors have been shown to attenuate the graft lesion, but the treatment of the receptor has had detrimental consequences. None of these agents were effective in reducing mortality rate, liver failure or perioperative morbidity in clinical studies; thus, not routinely or in liver transplantation protocols. Although multivariate and pleiotropic strategies have been considered, few experiments have shown positive results, which may contraindicate cocktails as pharmacological therapy (Figure 3 ). In this sense, the degree of IR injury influences the types of drugs of choice. Therefore, the duration and form of ischemia, as well as the inherent characteristics of the liver, should be taken into account in the studies. Moreover, considering the unpredictability of the biomarkers involved in the complex pathophysiology of the lesion, it is necessary that possible additional or synergistic effects be evaluated ${ }^{35-36}$.

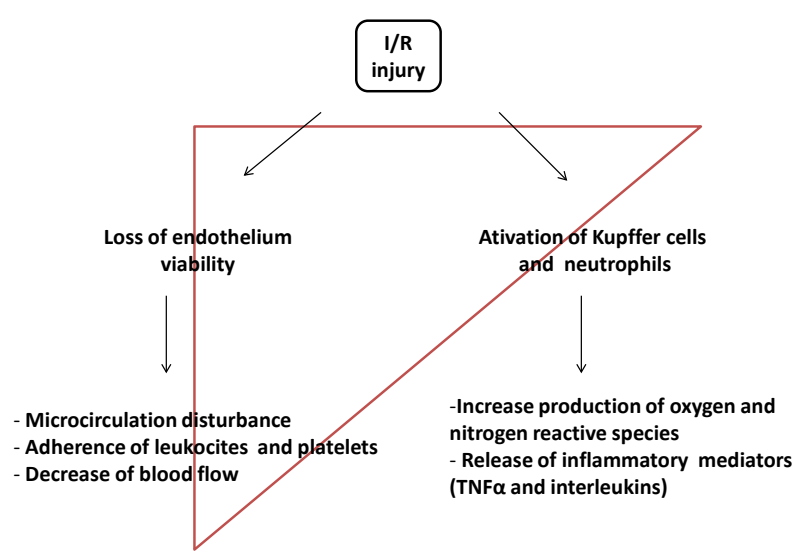

Figure 3 - Inflammatory process involved during the IR Injury, showing the consequences of endothelial loss function and activation of Kupffer cells and neutrophils in the liver.

\section{Other emerging options}

\section{Gene therapy}

As for gene therapy, the search for strategies in genes and molecular factors involved with the progression of the $\mathrm{I} / \mathrm{R}$ lesion grows. Depletion of NF-KB receptors denoted the use of IL-10 suppresses the cascade of NF-KB, increases the expression of hemeoxygenase-1, an enzyme of potent antiinflammatory effects. We have already tested models of suppression of the RNA translator of ICAM-, suppression of RNA responsible for TNF $\alpha$; blockers of Interleukin-1 Receptors, all of which are effective in hepatic protection ${ }^{37}$. 


\section{Traditional chinese medicine}

Alternatively, Traditional Chinese Medicine medications have been studied and have high efficacy. Paeoniflorin is a potent immunosuppressant, while trans-resveratrol alters the TLR4/NF-kB signaling pathway, decreasing the production of cytokines. Tetrandrine is a calcium channel blocker and decreases oxidative stress, while astaxanthin acts by reducing xanthine oxidase. Basically, the protective effect of the compounds used in Traditional Chinese Medicine comes from immunosuppressive effects, resistance to calcium influx and interferences in energy metabolism ${ }^{38}$.

\section{Hyperbaric oxygen}

Recently, our group has been working with various forms of conditioning. Preconditioning with oxygen in a hyperbaric chamber presented controversial results about mitochondrial function, evaluated through state 3; some studies did not identify a significant difference while others identified time-related improvement within the hyperbaric chamber. However, the preservation of mitochondrial membrane integrity and hepatocellular protection, measured by State 4 and hepatic enzymes, are congruent in the studies ${ }^{39-40}$.

\section{Laser therapy}

Laser therapy is already established in certain areas of medicine, such as cancer therapy, and its stabilizing effect on cell membranes is already well known, which gives it the ability to optimize liver regeneration after hepatectomies. Studies have described the relationship between wavelength and its effect on oxidative stress in animals not submitted to operative or chemical interventions, finding little or no difference between groups of different wavelengths. In general, the laser obtained positive results in the tangent to the reduction of oxidative stress ${ }^{41-43}$. Its use is then focused on hepatocellular protection during the IR lesion.

\section{Methylene blue}

Also, a case of the clinicaluse of methylene blue for reversion of vasopregic disease with signs of organ dysfunction and hypovolemic shock after elective liver transplantation has been reported. Although the practice was not routine, the situation was controlled, consonant with the existing literature ${ }^{44-45}$.

\section{Concluding remarks}

The main topics included on this review are summarized on Chart 1.

Chart 1 - Liver ischemia and reperfusion injury and its pathophysiology aspects in relation to the preconditioning: a simplified overview of the past, present and future.

- It was believed in the past that the flow interruption should not exceed 20 minutes, which limited the use of this maneuver. However, in subsequent studies, it has been postulated that ischemia could be maintained for more than 60 minutes in healthy livers.

- The pathophysiology liver IR includes three stages of the liver ischemia-reperfusion injury and an innate and acquired immunity.

- Oxidative stress, apoptosis and autophagy are detrimental injury mechanisms.

- Some important biomarkers (Tumor Necrosis Factor- $\alpha$, nitric oxide, metalloproteinases) have pivotal role.

- Preventive ("cheating") strategies, non-pharmacological and pharmacological options to treat the liver IR injury.

- To mitigate the lesion caused and prevent organ loss, various strategies have been studied, from surgical interventions, pharmacological agents to gene therapy. 


\section{References}

1. Freitas $S H$, Dória RG, Bueno RS, Rocha WB, Jair Filho RE, Moraes JR, Vidane AS, Ambrósio CE. Evaluation of potential changes in liver and lung tissue of rats in an ischemia-reperfusion injury model (modified pringle maneuver). PLoS One. 2017;12(6):e0178665. doi: 10.1371/journal. pone.0178665.

2. Gracia-Sancho J, Casillas-Ramírez A, Peralta C. Molecular pathways in protecting the liver from ischaemia/reperfusion injury: a 2015 update. Clin Sci (Lond). 2015;129(4):345-62. doi: 10.1042/CS20150223.

3. van Riel WG, van Golen RF, Reiniers MJ, Heger M, van Gulik TM. How much ischemia can the liver tolerate during resection? Hepatobiliary Surg Nutr. 2016;5(1):58. doi: 10.3978/j.issn.2304-3881.2015.07.05.

4. Karatzas T, Neri AA, Baibaki ME, Dontas IA. Rodent models of hepatic ischemiareperfusion injury: time and percentagerelated pathophysiological mechanisms. J Surg Res. 2014;191(2):399-412. doi: 10.1016/j.jss.2014.06.024.

5. Chu MJ, Hickey AJ, Phillips AR, Bartlett AS. The impact of hepatic steatosis on hepatic ischemia-reperfusion injury in experimental studies: a systematic review. Biomed Res Int. 2013;2013:192029. doi: 10.1155/2013/192029.

6. Tashiro H, Kuroda S, Mikuriya Y, Ohdan H. Ischemia-reperfusion injury in patients with fatty liver and the clinical impact of steatotic liver on hepatic surgery. Surg Today. 2014;44(9):1611-25. doi: 10.1007/ s00595-013-0736-9.

7. Nastos C, Kalimeris K, Papoutsidakis N, Tasoulis MK, Lykoudis PM, Theodoraki K, Nastou D, Smyrniotis V, Arkadopoulos N. Global consequences of liver ischemia/reperfusion injury. Oxid Med Cell Longev. 2014;2014:906965. doi: $10.1155 / 2014 / 906965$.

8. Cannistrà M, Ruggiero $M$, Zullo A, Gallelli, G, Serafini S, Maria M, Naso A, Grande R, Serra $R$, Nardo B. Hepatic ischemia reperfusion injury: a systematic review of literature and the role of current drugs and biomarkers. Int J Surg. 2016;33:S57-S70. doi: 10.1016/j. ijsu.2016.05.050.

9. Guan LY, Fu PY, Li PD, Li ZN, Liu HY, Xin MG,
Li W. Mechanisms of hepatic ischemiareperfusion injury and protective effects of nitric oxide. World J Gastrointest Surg. 2014;6(7):122. doi: 10.4240/wjgs.v6.i7.122.

10.Peralta C, Jiménez-Castro MB, GraciaSancho J. Hepatic ischemia and reperfusion injury: effects on the liver sinusoidal milieu.

J Hepatol. 2013;59(5):1094-106. doi: 10.1016/j.jhep.2013.06.017.

11.Datta G, Fuller BJ, Davidson BR. Molecular mechanisms of liver ischemia reperfusion injury: insights from transgenic knockout models. World J Gastroenterol. 2013;19(11):1683. doi: 10.3748/wjg.v19. i11.1683.

12.Chang WJ, Toledo-Pereyra LH. Toll-like receptor signaling in liver ischemia and reperfusion. J Invest Surg. 2012;25(4):2717. doi: 10.3109/08941939.2012.687802.

13.Lu L, Zhou H, Ni M, Wang X, Busuttil R, Kupiec-Weglinski J, Zhai Y. Innate immune regulations and liver ischemia reperfusion injury. Transplantation. 2016;100(12):2601. doi: 10.1097/TP.0000000000001411

14.van Golen RF, Reiniers MJ, Vrisekoop N, Zuurbier CJ, Olthof PB, van Rheenen J, van Gulik TM, Parsons BJ, Heger M. The mechanisms and physiological relevance of glycocalyx degradation in hepatic ischemia/ reperfusion injury. Antioxid Redox Signal. 2014;21(7):1098-118. doi: 10.1089/ ars.2013.5751.

15.Zimmerman MA, Martin A, Yee J, Schiller J, Hong JC. Natural killer T cells in liver ischemia-reperfusion injury. J Clin Med. 2017;6(4):41. doi: 10.3390/jcm6040041.

16. Elias-Miro $M$, Jiménez-Castro $M B$, Rodés J, Peralta C. Current knowledge on oxidative stress in hepatic ischemia/reperfusion. Free Radic Res. 2013;47(8):555-68. doi: 10.3109/10715762.2013.811721.

17. Reiniers MJ, van Golen RF, van Gulik TM, Heger $M$. Reactive oxygen and nitrogen species in steatotic hepatocytes: a molecular perspective on the pathophysiology of ischemia-reperfusion injury in the fatty liver. Antioxid Redox Signal. 2014;21(7):1119-42. doi: 10.1089/ars.2013.5486.

18.Jones RT, Toledo-Pereyra LH, Quesnelle KM. Selectins in liver ischemia and reperfusion injury. J Invest Surg. 2015;28(5):292-300. doi: 10.3109/08941939.2015.1056920.

19.Shuh $M$, Bohorquez $H$, Loss Jr GE, 
Cohen AJ. Tumor necrosis factor- $\alpha$ : life and death of hepatocytes during liver ischemia/reperfusion injury. Ochsner J. 2013;13(1):119-30. PMID: 3603175.

20.Zhang YQ, Ding N, Zeng YF, Xiang YY, Yang MW, Hong FF, Yang SL. New progress in roles of nitric oxide during hepatic ischemia reperfusion injury. World J Gastroenterol. 2017;23(14):2505. doi: 10.3748/wjg.v23. i14.2505.

21.Palladini G, Ferrigno A, Richelmi P, Perlini S, Vairetti M. Role of matrix metalloproteinases in cholestasis and hepatic ischemia/ reperfusion injury: a review. World J Gastroenterol. 2015;21(42):12114. doi: 10.3748/wjg.v21.i42.12114.

22.Quesnelle KM, Bystrom PV, Toledo-Pereyra LH. Molecular responses to ischemia and reperfusion in the liver. Arch Toxicol. 2015;89(5):651-7. doi: 10.1007/s00204014-1437-x.

23.Cursio R, Colosetti P, Gugenheim J. Autophagy and liver ischemia-reperfusion injury. Biomed Res Int. 2015; 2015:417590. doi: 10.1155/2015/417590.

24.de Lourdes Jorge G, dos Reis Tártaro R, Escanhoela CF, Boin IFSF. Later evaluation of ischemia and reperfusion by the pringle maneuver in Wistar rats, demonstrating that hepatic lesions can be reversible. Transplant Proc. 2017;49(4):898-901. doi: 10.1016/j. transproceed.2017.01.054.

25.Li J, Li RJ, Lv GY, Liu HQ. The mechanisms and strategies to protect from hepatic ischemiareperfusion injury. Eur Rev Med Pharmacol Sci. 2015;19(11):2036-47. PMID: 26125267.

26.Saidi RF, Kenari SK. H. Liver ischemia/ reperfusion injury: an overview. J Invest Surg. 2014;27(6):366-79. doi: 10.3109/08941939.2014.932473.

27.Zimmerman MA, Kam I, Eltzschig $H$, Grenz A. Biological implications of extracellular adenosine in hepatic ischemia and reperfusion injury. Am J Transplant. 2013;13(10):2524-9. doi: 10.1111/ ajt.12398.

28.Centurion SAR, Centurion LM, Souza MEJ, Gomes MCJ, Sankarankutty AK, Mente $E D$, Silva OC. Effects of ischemic liver preconditioning on hepatic ischemia/ reperfusion injury in the rat. Transplant Proc. 2007;39(2):361-4. doi: 10.1016/j. transproceed.2007.01.011.
29.Stokfisz K, Ledakowicz-Polak A, Zagorski M, Zielinska M. Ischaemic preconditioningCurrent knowledge and potential future applications after 30 years of experience. Adv Med Sci. 2017;62(2):307-16. doi: 10.1016/j.advms.2016.11.006.

30.Pacheco EG, Gomes MCJ, Rodrigues GR, Campos WKR, Castro e Silva O. Efeito do pré-condicionamento isquêmico hepático submetidos a lesão de isquemia/reperfusão do fígado. Acta Cir Bras. 2006;21(1):24-8.

31.Chu MJ, Vather R, Hickey AJ, Phillips AR, Bartlett AS. Impact of ischemic preconditioning on outcome in clinical liver surgery: a systematic review. Biomed Res Int. 2015; 2015:370451. doi: 10.1155/2015/370451.

32. Robertson FP, Magill LJ, Wright GP, Fuller B, Davidson BR. A systematic review and metaanalysis of donor ischaemic preconditioning in liver transplantation. Transpl Int. 2016;29(11):1147-54. doi: 10.1111/ tri.12849.

33. Guo Y, Feng L, Zhou Y, Sheng J, Long D, Li S, Li Y. Systematic review with meta-analysis: HIF$1 \alpha$ attenuates liver ischemia-reperfusion injury. Transplant Rev. 2015;29(3):127-34. doi: 10.1016/j.trre.2015.05.001.

34. Theodoraki K, Karmaniolou I, Tympa A, Tasoulis MK, Nastos C, Vassiliou I, Arkadopoulos N, Smyrniotis V. Beyond preconditioning: postconditioning as an alternative technique in the prevention of liver ischemia-reperfusion injury. Oxid Med Cell Longev. 2016; 2016:8235921. doi: 10.1155/2016/8235921.

35.Akhtar MZ, Henderson T, Sutherland A, Vogel T, Friend PJ. Novel approaches to preventing ischemia-reperfusion injury during liver transplantation. Transplant Proc. 2013;45(6):2083-92. doi: 10.1016/j. transproceed.2013.04.004.

36. Yamanaka K, Houben P, Bruns H, Schultze D, Hatano E, Schemmer P. A systematic review of pharmacological treatment options used to reduce ischemia reperfusion injury in rat liver transplantation. PloS One. $2015 \mathrm{Apr}$ 28;10(4):e0122214. doi: 10.1371/journal. pone. 0122214.

37.Suyavaran A, Thirunavukkarasu C. Preconditioningmethodsinthemanagement of hepatic ischemia reperfusion-induced injury: Update on molecular and future 
perspectives. Hepatol Res. 2017;47(1):3148. doi: 10.1111/hepr.12706.

38. Hu C, Li L. Pre-conditions for eliminating mitochondrial dysfunction and maintaining liver function after hepatic ischaemia reperfusion. J Cell Mol Med. 2017 Sep;21(9):1719-31. doi: 10.1111/ jcmm.13129.

39.Baldim LB, Nejo Jr R, Souza MEJ, Gomes MCJ, Picinato MANC, Fina CF, Castro-e-Silva $O$. Effect of hyperbaric oxygen therapy on liver function during intermittent ischemia. Acta Cir Bras. 2013;28:61-5. PMID: 23381826.

40.Losada DM, Jordani ME, Jordani MC, Piccinato MANC, Fina CF, Feres O, Chies AB, Evora PR, Castro e Silva $O$. Should preconditioning hyperbaric oxygenation protect the liver against ischemia-reperfusion injury? An experimental study in a rat model. Transplant Proc. 2014;46(1):56-62. doi: 10.1016/j.transproceed.2013.10.044.

41.Bagnato VS, Kurachi C, Ferreira J, Sankarankutty AK, Zucoloto S, Castro e Silva O. New photonic technologies for the treatment and diagnosis of hepatic diseases: an overview of the experimental work performed in collaboration, between Physics Institute of Sao Carlos and Ribeirao Preto Faculty of Medicine of the University of Sao Paulo. Acta Cir Bras. 2006;21 Suppl
1:3-11. PMID: 17013504.

42.Barbosa AJ, Santana ACDA, Castro e Silva T, Kurachi C, Inada N, Bagnato VS. Effect of laser on the remnant liver after the first 24 hours following $70 \%$ hepatectomy in rats. Acta Cir Bras. 2011;26(6):470-4. PMID: 22042110.

43. Mumic FT, Silveira MRGD, Vilalva KH, Jordani ME, Gomes MCJ, Vanni JC, Vollet Filho JD, Kurachi C, Bagnato VS, Silva OC. Effect of irradiation with different laser wavelengths on oxidative stress of non-hepatectomized rats. Acta Cir Bras. 2016;31:40-4. doi: 10.1590/S0102-86502016001300009.

44. Rosique R, Rosique MJF, Rosique IA, Tirapelli LF, Silva OC, dos Santos JS, Evora PRB. Effect of methylene blue on the hemodynamic instability resulting from liver ischemia and reperfusion in rabbits. Transplant Proc. 2011;43(10):3643-51. doi: 10.1016/j. transproceed.2011.08.108.

45.Vilalva KH, Mumic FT, Silveira MR, Mente ED, Evora PR, Castro ESO. Use of methylene blue to treat hypovolemic shock followed by ischemia-reperfusion injury in the postoperative orthotopic liver transplant patient: a case report. Exp Clin Transplant. 2018 Aug;16(4):511-4. doi: 10.6002/ ect.2016.0041.

\section{Correspondence:}

Orlando Castro-e-Silva

Departamento de Cirurgia e Anatomia, USP

orlando@fmrp.usp.br
Conflict of interest: none

Financial source: none
Received: Apr 11, 2018

Review: June 12, 2018

Accepted: July 15, 2018
${ }^{1}$ Research performed at Department of Surgery and Anatomy, Medical School, Universidade de São Paulo (USP), Ribeirao Preto-SP, Brazil. 